\title{
Partial visual recovery from radiation-induced optic neuropathy after hyperbaric oxygen therapy in a patient with Cushing disease
}

\author{
Mara Boschetti ${ }^{1}$, Massimo De Lucchi ${ }^{2}$, Massimo Giusti ${ }^{1}$, Claudio Spena ${ }^{2}$, Guido Corallo ${ }^{3}$, Umberto Goglia ${ }^{1}$, \\ Enrica Ceresola ${ }^{1}$, Eugenia Resmini ${ }^{1}$, Lara Vera ${ }^{1}$, Francesco Minuto ${ }^{1}$ and Diego Ferone ${ }^{1}$ \\ ${ }^{1}$ Department of Endocrinological and Metabolic Sciences and Center of Excellence for Biomedical Research, ${ }^{2}$ Department of Anesthesiology, Unit of \\ Hyperbaric Therapy and ${ }^{3}$ Institute of Ophthalmology, University of Genoa and San Martino Hospital, Viale Benedetto XV, 6, I-16132, Genoa, Italy \\ (Correspondence should be addressed to D Ferone; Email: ferone@unige.it)
}

\begin{abstract}
Here we describe the case of a 41-year-old woman with a history of Cushing disease who had previously undergone unsuccessful neurosurgery, followed by stereotactic radiosurgery. More than 4 years after this treatment, she presented severe visual impairment, which started in the left eye and was documented by neuro-ophthalmic evaluation. Radiological assessment by contrast-enhanced magnetic resonance (MR) imaging initially suggested the diagnosis of glioma of the optic nerve and the patient started corticosteroid treatment (first with prednisone, $80 \mathrm{mg} /$ day, followed by dexamethasone, $8 \mathrm{mg} /$ day). Despite the therapy, vision in the left eye rapidly worsened until light was no longer perceptible; similar symptoms and signs also developed in the right eye, evolving to complete temporal hemianopsia. The clinical evidence was confirmed by the rapid progression of the MR picture, which showed homogeneous enhancement of the chiasm and optic nerves. On the basis of these findings, the original diagnosis of glioma was excluded, and radiation-induced optic neuropathy was diagnosed. As corticosteroids had proved inefficacious, hyperbaric oxygen (HBO) therapy was promptly instituted and vision steadily started to improve. This improvement was documented and confirmed by the progressive recovery of the visual field in the right eye and the changes in the sequential follow-up MR scanning. Optic neuropathy is an infrequent but dramatic complication of radiation therapy. Symptoms develop, on average, 12 months after treatment, and the onset may be acute and characterized by the progressive loss of vision in one or both eyes. HBO has already been used to treat this complication, but its efficacy is still controversial. Here, in addition to describing this particular case, which presented a significantly delayed radiation injury of the optic pathways, we provide a brief literature review and discuss some important points.
\end{abstract}

European Journal of Endocrinology 154 813-818

\section{Introduction}

For many years, radiotherapy has been used to treat pituitary adenomas after failure of surgery. Despite a maximal 45-55 Gy dose with optimal standard daily fractionation of 1.8-2 Gy, a few serious complications (i.e. temporal brain necrosis and optic neuropathy) have occurred; consequently, endocrinologists and neurosurgeons have tended to limit the use of this approach (1). However, the last decade has seen important advances in radiotherapy technology which combine precise tumor localization with accurately targeted delivery of radiation. This technique of high-precision conformal radiotherapy, described as stereotactic radiotherapy or radiosurgery, uses modern linear accelerators available in most radiation departments, and has renewed the role of radiation therapy in pituitary adenomas $(1,2)$.

Characterized by abrupt and permanent visual loss, optic neuropathy is a recognized but infrequent dramatic complication of radiation therapy. Symptoms develop, on average, 12 months after conventional treatment; the onset may be acute and is followed by the progressive loss of vision in one or both eyes, to the point of total blindness $(3,4)$. This complication generally occurs after cumulative doses of radiation exceeding 50 Gy, or single doses greater than 10 Gy delivered to the visual apparatus. Visual loss may result from lesions of the disc, the retrobulbar segment of the optic nerve, optic chiasm, or even the retrogeniculate pathways (3, 4). The second eye may develop abnormalities and clinical manifestations of optic neuropathy from days to months after the diagnosis in the first eye involved.

Various treatments, including corticosteroids, have generally failed to reverse or even halt the loss of vision due to radiation injury of visual pathways (4). Hyperbaric oxygen (HBO) therapy has also been used to treat this complication, but its efficacy is still controversial. We report the case of a patient who 
presented with extremely delayed radiation injury to the optic pathways 4 years after stereotactic radiosurgery for persistent Cushing disease. HBO partially reversed visual loss in the more recently involved eye, but was unsuccessful in the first eye affected.

\section{Case report}

A few weeks before admission, a 41-year-old woman noticed blurred and decreased vision in the left eye. This was documented by the partial deficit of the superior and temporal quadrants of the visual field; the visual field in the right eye was normal. There were no other symptoms and she had normal visual acuity, color vision and pupil. Ophthalmoscopy was normal in the right eye, whereas in the left eye the optic disc appeared pale. Magnetic resonance (MR) showed an enlarged left optic nerve and left hemichiasm, which enhanced after gadolinium injection (Fig. 1A), while fluorine-18 fluorodesoxyglucose $\left({ }^{18} \mathrm{~F}\right.$-FDG) positron emission tomography (PET) displayed a scant local pathological accumulation of the tracer. Patient history revealed that more than 4 years before admission she had undergone unsuccessful neurosurgery for Cushing disease, followed by stereotactic radiosurgery (LINAC 2010). Single dose planning had been carried out using thinsection axial computed tomography with reformatted coronal images, and consisted of one isocenter located centrally in the residual pituitary adenoma, yielding a maximal dose of $20 \mathrm{~Gy}$; the dose to the tumor margin was $10 \mathrm{~Gy}$. From these data, the dose of radiation to the optic nerve and chiasm was calculated to have been approximately $8 \mathrm{~Gy}$. In this patient, before and during the follow-up, vision, visual fields and MR features were normal until visual symptoms developed 4 years after radiation treatment.

On admission, the levels of plasma cortisol and adrenocorticotropin (ACTH) and urinary free cortisol were within the normal range. Full endocrinological evaluation revealed normal pituitary, thyroid and gonadal function. On the basis of her clinical history and the radiological and nuclear medicine imaging findings, radiation-induced glioma was strongly suggested by radiologists, although delayed necrosis of the optic nerve and chiasm could not be ruled out. Conversely, other potential causes of optic nerve neuritis, such as Lyme disease, tuberculosis, syphilis, viral agents (HIV, HBV, herpes virus, cytomegalovirus), as well as secondary involvement from infection of the paranasal sinus or orbits, were excluded on the basis of clinical evidences, history and appropriate tests. The patient began receiving oral prednisone ( $80 \mathrm{mg} /$ day), which was tapered over 15 days. Despite this therapy, vision in the left eye rapidly worsened, and within 2 weeks light was no longer perceptible. Neuro-ophthalmic examination confirmed a rapid visual deterioration in the right eye: the optic disc became pale and a slight
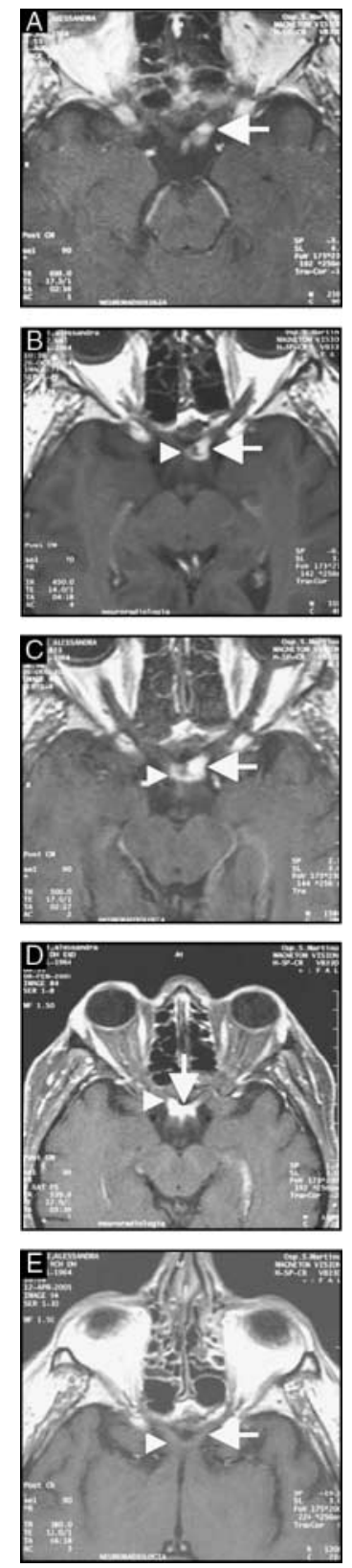

Figure 1 Gadolinium-enhanced axial magnetic resonance imaging scans before and during treatment with corticosteroids $(A-D)$ and after hyperbaric oxygen therapy $(E)$. (A) Baseline scan shows swelling and enhancement of the left optic nerve (arrow) and its junction with the left side of the chiasm; $(B)$ on day 15 , the scan shows further development of the previous picture (arrow), and an initial involvement of the right side of the chiasm (arrowhead); (C) on day 45, the scan shows the high signal indicating the complete involvement of the left optic nerve and hemichiasm (arrow) and the further development of the enhancement and swelling of the right hemichiasm (arrowhead); (D) on day 60, the scan shows enhancement within the entire area of the chiasm (arrow) and the involvement of the right optic nerve (arrowhead); (E) on day 90, the scan shows atrophy of the left optic nerve and chiasm (arrow), and swelling, but no evidence of enhancement, of the right optic nerve (arrowhead). 

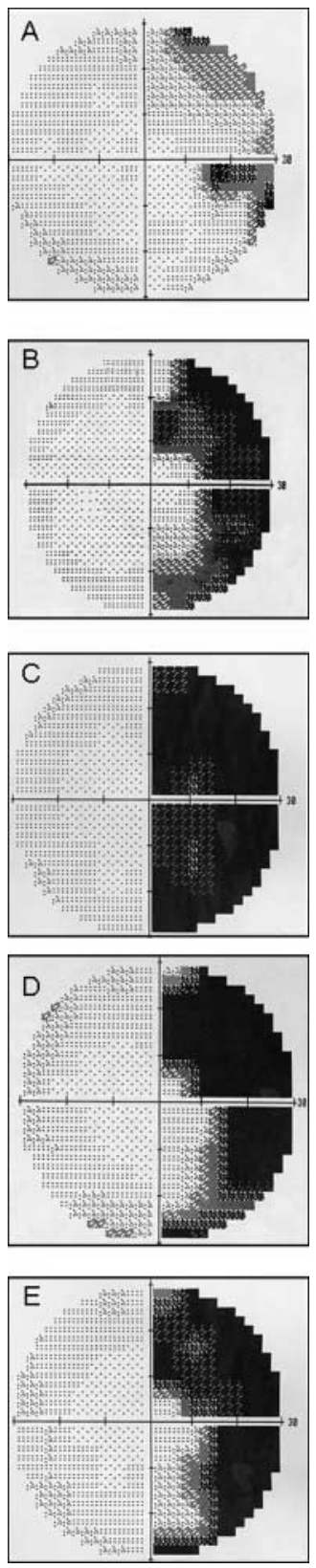

Figure 2 Visual fields in the right eye before and during treatment with dexamethasone $(\mathrm{A}-\mathrm{C})$ and before and after hyperbaric oxygen therapy (D-E). Automated static threshold perimetry (Humphrey 750 II VFA, program 30-2) shows: (A) some defects in the temporal quadrants of the visual field; (B) almost complete temporal hemianopia; (C) complete temporal hemianopia; (D, E) partial, but significant, recovery of the temporal hemianopia.

visual field impairment was detected (Fig. 2A). Repeat contrast-enhanced MR scanning showed persistent swelling and enhancement of the left optic nerve and hemichiasm, and initial involvement of the right side of the chiasm (Fig. 1B). Corticosteroid treatment was therefore restarted. However, despite dexamethasone therapy $(8 \mathrm{mg} /$ day $)$ tapered over 1 month, vision deteriorated to almost complete temporal hemianopia in the right eye (Fig. 2B). Meanwhile, MR showed further enlargement of the chiasm and optic nerves (Fig. 1C). This picture quickly worsened over the next 15 days, evolving to complete temporal hemianopia in the right eye (Fig. 2C), while MR showed homogeneous enhancement of the chiasm and optic nerves (Fig. 1D). Given the very rapid progression of the visual and radiological findings, the original diagnosis of glioma was questioned, and a diagnosis of radiation-induced optic neuropathy appeared more credible. After written informed consent had been given by the patient, HBO therapy was promptly instituted and after 15 days vision started to improve steadily. The clinical improvement was documented and confirmed by the progressive, albeit partial, recovery of the visual field in the right eye, observed on perimetry during the HBO cycle (Fig. 2D, E). Conversely, the lack of light perception persisted in the left eye. Optic nerve and chiasm enhancement was no longer evident on follow-up MR scanning performed after completion of the HBO therapy. However, atrophy of the left optic nerve and the related portion of the chiasm was observed (Fig. 1E). The clinical trend of the visual field was evaluated by monitoring the mean defect (MD) and the pattern S.D. (PSD) indexes. The MD and the PSD trend during dexamethasone therapy, HBO treatment and during the subsequent follow-up period is represented graphically in Fig. 3.

\section{Methods}

\section{Magnetic resonance imaging}

Sellar and perisellar regions, chiasm and optic nerves were evaluated by MR performed on clinical $1 \mathrm{~T}$ scanners, using $\mathrm{T} 1$ and $\mathrm{T} 2 \mathrm{~S}$ weighted-gradient recalled-echo (repetition time, 200-300 ms; echo time, $10-12 \mathrm{~ms}$; flip angle, $90^{\circ}$; four signal averages) in the axial, sagittal and coronal planes. Acquisitions were carried out before and after the administration of gadolinium chelate $(0.1 \mathrm{mmol})$. MR was performed at the baseline and 15, 45, 60 and 90 days after admission.

\section{Visual perimetry}

Visual field was evaluated by means of a Humphrey perimeter (750 II VFA model, program 'central 30-2', full threshold strategy). In particular, indexes of automatic static perimetry, namely MD and PSD, were evaluated. The MD displays the reduction of mean sensibility of visual field compared with normal sensibility for the same age of patient. The PSD shows how the shape of the visual field differs from the normal shape for the same age of patient.The ophthalmological examination was performed at the same intervals as the 


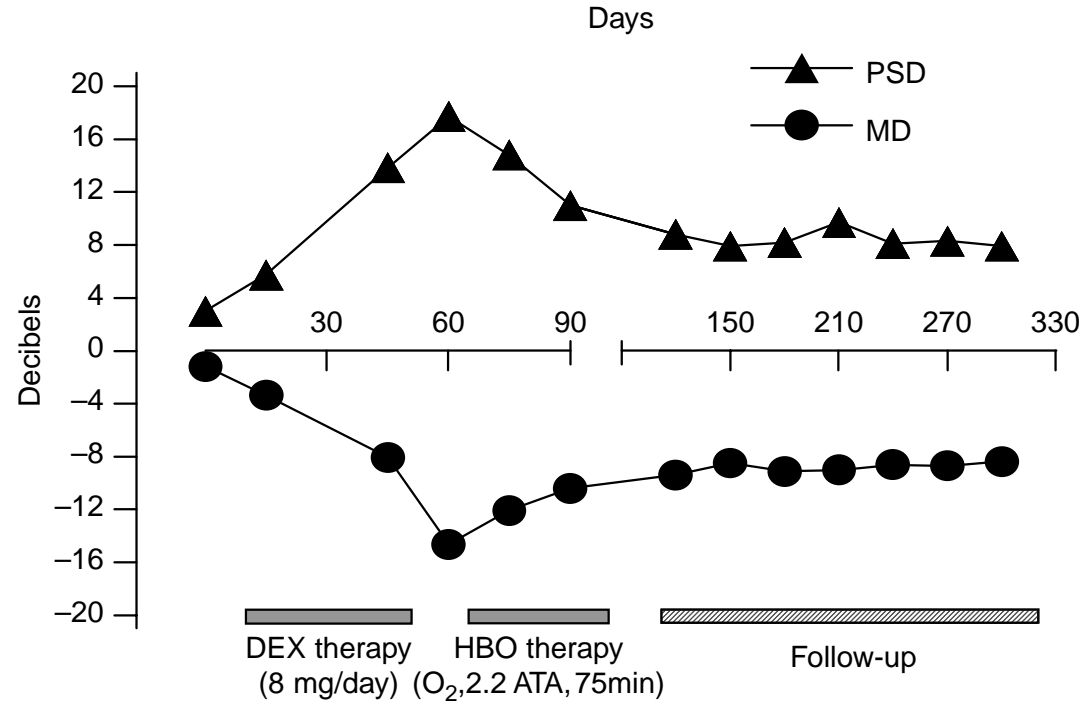

Figure 3 Visual field parameters during the sequential evaluation from days 1 to 300 . Horizontal bars indicate period of dexamethasone (DEX) administration and hyperbaric oxygen (HBO) therapy. MD, mean defect; PSD, pattern S.D.
MR and every 15 days during HBO therapy, then every 30 days for the remaining follow-up period.

\section{Hyperbaric oxygen therapy}

HBO is the inhalation of $100 \%$ oxygen inside a hyperbaric chamber pressurized to greater than 1 atm. HBO was scheduled at 2.2 ATA (absolute atmospheric pressure, $220 \mathrm{kPa}$ ) 5 days per week for 41 days. Each session was held in a hyperbaric multiplace chamber (Sistemi Iperbarici Integrati, Italy) and lasted $75 \mathrm{~min}$ (10 min to reach 2.2 ATA, followed by two cycles of $30 \mathrm{~min}$ hyperbaric $100 \%$ oxygen through a mask, separated by $5 \mathrm{~min}$ of hyperbaric normal air from the chamber), during which $100 \%$ oxygen was inhaled through a facemask (Airtech, Cressi, Italy).

\section{Discussion}

HBO therapy yielded partial visual recovery in a patient with radiation-induced optic neuropathy that occurred more than 4 years after stereotactic radiosurgery. In this patient, treatment with high doses of corticosteroids proved ineffective, and rapid progression of the visual abnormalities was observed before the institution of HBO therapy. Although a spontaneous improvement may also occur in patients with radiation-induced optic nerve neuritis, the quick improvement and the rapid progressive changes in the radiological pictures during HBO therapy strongly support its role in the partial regression of the ocular symptoms.

Radiation injury of the optic nerves and chiasm is a well-documented complication of conventional external beam radiation therapy. It may occur months to years after completion of treatment. However, in $90 \%$ of cases it appears within 3 years of irradiation, with a mean onset at 12 months (3-5). Visual loss generally arises in one eye and is acute in onset. The degree of visual loss is severe and may progress to total blindness. The availability of stereotactic radiosurgery and the gamma knife have significantly reduced the occurrence of this severe side-effect of radiation treatment $(1,2)$. However, the clinical course is similar to that observed after conventional radiotherapy, and with the increase in the number of radiosurgical centers, this complication may occur more frequently. A recent study evaluated the risk of radiation-induced optic neuropathy in a large series of patients (215) with benign tumors adjacent to the optic apparatus, including 89 pituitary adenomas, who had undergone stereotactic radiosurgery (6). Radiation optic neuropathy occurred in less than $2 \%$ of patients, despite the fact that the majority received more than 8 Gy to the optic area, a dose which, although often questioned (7-9), is still regarded as the recommended maximum (6). Interestingly, of the four patients who developed radiation optic neuropathy, three had Cushing disease. The calculated dose to optic structures in these three patients ranged from 9 to $12.8 \mathrm{~Gy}$, and the time of onset ranged from 18 to 36 months. However, it should be pointed out that two of these patients had previously received external beam radiotherapy and one presented pre-treatment visual defects (6). Both of these conditions are regarded as risk factors for the development of radiation optic neuropathy after stereotactic radiosurgery. Previously, however, Girkin and co-workers reported on four patients who underwent stereotactic radiosurgery; in this small series, too, one patient with Cushing disease received a dose to the optic nerves ranging from 7 to 14 Gy and developed optic neuropathy 16 months after treatment (10). Our patient also underwent stereotactic radiosurgery for persistent Cushing disease after unsuccessful neurosurgery. To our knowledge, however, 
this is the first case of pituitary adenoma in which optic radionecrosis developed more than 48 months after treatment with a relatively low dose of radiation. This is one of the reasons why the case was initially misdiagnosed. Nevertheless, it should be emphasized that radionecrosis of the anterior visual pathways is a diagnosis by exclusion, requiring careful ophthalmologic testing and sequential gadolinium-enhanced MR scanning. Radiation-induced lesions in the anterior visual pathway typically cannot be demonstrated on computerized tomographic scans. Contrasted MR enhancement is the preferred diagnostic method. Damaged neural tissue shows enhancement and swelling. The enhancement fades within 3 months of symptom onset as atrophy appears (4). The question arises as to whether the long-term condition of hypercortisolism might be involved in the pathogenesis of delayed radiation-induced optic neuropathy. Careful retrospective studies should be carried out to shed light on this issue and to help decide the most appropriate therapeutic strategy in these already complicated cases. Indeed, stereotactic radiosurgery has recently been tentatively suggested as the first-line treatment for patients with Cushing disease (11).

A recent review of treatments of radiation injury to the visual pathways has shown a rather dramatic picture of unsuccessful results (4). With few exceptions, treatment with corticosteroids has been unsuccessful, whereas other medical treatments have proved completely ineffective (4). Conversely, HBO therapy, though still controversial, seems to be more promising than any other form of treatment (4). HBO therapy involves the inhalation of $100 \%$ oxygen at a pressure greater than 1 atm absolute $(100 \mathrm{kPa})$. It uses oxygen as a drug by dissolving it in the plasma and delivering it to the tissues independently of hemoglobin. For a variety of organ systems, HBO is known to promote new vessel growth in areas with reduced oxygen tension due to poor vascularization, and therewith promotes wound healing and recovery of radiationinjured tissue (12). Guy and Schatz first advocated the use of early HBO therapy in patients with radiationinduced optic neuropathy (13). Later, in their review of published cases, Borruat et al. compared visual outcomes among untreated patients and patients treated with two regimens of HBO therapy (HBO 2.0 ATA, and $\mathrm{HBO} \geq 2.4$ ATA) and suggested that HBO was more effective at higher atmospheric pressures (5). Pasquier et al. recently reviewed the role of HBO therapy in radio-induced lesions in normal tissues (14). Having evaluated the few retrospective studies available in the literature and focused on optic neuropathy, these authors concluded that although a minority of patients displayed a significant improvement in vision as a result of HBO therapy, few of these regained normal eyesight (14). Moreover, other authors have suggested the importance of early treatment and explain the failure of $\mathrm{HBO}$ in some studies as a consequence of delay in starting the treatment (15).

Since HBO is effective only if delivered soon after the onset of symptoms, efforts should be made to detect anterior visual pathway radiation injury as early as possible. Some authors have noted that patients may have MR signs of radiation injury that antedate the loss of vision (4). Therefore, in patients undergoing radiation therapy, including stereotactic radiosurgery, frequent enhanced MR should be performed during the period of highest risk. In fact, although the occurrence of this side-effect is rare, the consequences are extremely serious. Because both eyes are often involved serially, similar testing should be carried out after one eye has developed radiation neuropathy, and if MR scanning reveals signs of radionecrosis, HBO treatment should also be given prophylactically. Moreover, visual evoked potential (VEP) may be abnormal months before the loss of vision (7). Kellner et al. evaluated VEP in patients with normal vision who were irradiated for uveal melanomas (16). Five patients had radiation papillopathy, and all of these also had abnormal VEP latency. In several cases, VEP was abnormal even when visual function and fundus examination were still normal. These findings suggest that serial testing of VEPs during the high-risk period in appropriate patients might provide early evidence of optic neuropathy and allow optimal application of HBO therapy.

In our patient, who had extremely delayed radiation injury to the optic nerves and chiasm, high doses of corticosteroids proved unsuccessful. By contrast, HBO therapy partially reversed visual loss in the more recently involved eye, although it was unsuccessful in the first eye affected. This particular case focuses attention to three important points. First, symptoms and signs of radiation injury to the optic nerves and chiasm may start later than previously reported, thus complicating differential diagnosis with a second tumor of the optic pathways. Moreover, optic radionecrosis may occur even after low radiation doses, as are generally required in patients with pituitary adenomas. The effects of radiation injury are difficult to reverse, since HBO treatment seems to repair only the damage produced within the last several days and not that which occurs weeks earlier. Secondly, hypercortisolism could be involved in the late onset, as well as in the higher prevalence, of this complication in patients with Cushing disease. Indeed, in a large series of patients with clinically non-functioning pituitary tumor who had undergone radiation therapy, no patient had radiation-induced optic neuropathy (17). Moreover, on reviewing the literature on radiation-treated nonfunctioning pituitary tumors, the same authors discovered that the incidence of radiation-induced optic neuropathy in these patients was $0.53 \%$ (17). Finally, the clinical course observed in our patient confirms that HBO therapy could be an effective, non-invasive modality for the treatment of radiation-induced optic neuropathy, 
and that prompt treatment and a proper technique are critical for the management of this severe complication.

\section{Acknowledgements}

This study was partially supported by grants from MIUR (2002067251-001) and from the University of Genoa.

\section{References}

1 Sheehan JP, Niranjan A, Sheehan JM, Jane JA Jr, Laws ER, Kondziolka D, Flickinger J, Landolt AM, Loeffler JS \& Lunsford LD. Stereotactic radiosurgery for pituitary adenomas: an intermediate review of its safety, efficacy, and role in the neurosurgical treatment armamentarium. Journal of Neurosurgery 2005102 678-691.

2 Ajithkumar T \& Brada M. Stereotactic linear accelerator radiotherapy for pituitary tumors. Treatments in endocrinology 20043 211-216.

3 Borruat F-X, Schatz NJ \& Glaser JS. Radiation optic neuropathy: report of cases, role of hyperbaric oxygen therapy and literature review. Neuro-Ophthalmology 199616 255-266.

4 Lessell S. Friendly fire: neurogenic visual loss from radiation therapy. Journal of Neuro-Ophthalmology $200424243-250$.

5 Borruat FX, Schatz NJ, Glaser JS, Feun LG \& Matos L. Visual recovery from radiation-induced optic neuropathy, The role of hyperbaric oxygen therapy. Journal of Clinical Neuroophthalmology $19931398-101$.

6 Stafford SL, Pollock BE, Leavitt JA, Foote RL, Brown PD, Link MJ, Gorman DA \& Schomberg PJ. A study on the radiation tolerance of the optic nerves and chiasm after stereotactic radiosurgery. International Journal of Radiation Oncology, Biology, Physics 2003 55 1177-1181.

7 Leber KA, Bergloff J \& Pendl G. Dose-response tolerance of the visual pathways and cranial nerves of the cavernous sinus to stereotactic radiosurgery. Journal of Neurosurgery $1998 \mathbf{8 8} 43-50$.
8 Morita A, Coffey RJ, Foote RL, Schiff D \& Gorman D. Risk of injury to cranial nerves after gamma knife radiosurgery for skull base meningiomas: experience in 88 patients. Journal of Neurosurgery $19999042-49$.

9 Ove R, Kelman S, Amin PP \& Chin LS. Preservation of visual fields after peri-sellar gamma-knife radiosurgery. International Journal of Cancer $200090343-350$.

10 Girkin CA, Comey CH, Lunsford LD, Goodman ML \& Kline LB. Radiation optic neuropathy after stereotactic radiosurgery. Ophthalmology $1997 \mathbf{1 0 4} 1634-1643$.

11 Hentschel SJ \& McCutcheon IE. Stereotactic radiosurgery for Cushing disease. Neurosurgical Focus 200416 E5.

12 Mayer R, Hamilton-Farrell MR, van der Kleij AJ, Schmutz J, Granstrom G, Sicko Z, Melamed Y, Carl UM, Hartmann KA, Jansen EC, Ditri L \& Sminia P. Hyperbaric oxygen and radiotherapy. Strahlentherapie und Onkologie 2005181 113-123.

13 Guy J \& Schatz NJ. Hyperbaric oxygen in the treatment of radiation-induced optic neuropathy. Ophthalmology 198693 1083-1088.

14 Pasquier D, Hoelscher T, Schmutz J, Dische S, Mathieu D, Baumann M \& Lartigau E. Hyperbaric oxygen therapy in the treatment of radio-induced lesions in normal tissues: a literature review. Radiotherapy and Oncology 200472 1-13.

15 Roden D, Bosley TM, Fowble B, Clark J, Savino PJ, Sergott RC \& Schatz NJ. Delayed radiation injury to the retrobulbar optic nerves and chiasm, Clinical syndrome and treatment with hyperbaric oxygen and corticosteroids. Ophthalmology 199097 346-351.

16 Kellner U, Bornfeld N \& Foerster MH. Radiation-induced optic neuropathy following brachytherapy for uveal melanomas. Graefe's Archive for Clinical and Experimental Ophthalmology 1993 231 267-270.

17 van den Bergh AC, Schoorl MA, Dullaart RP, van der Vliet AM, Szabo BG, ter Weeme CA \& Pott JW. Lack of radiation optic neuropathy in 72 patients treated for pituitary adenoma. Journal of Neuroophthalmology 200424 200-205.

Received 25 October 2005

Accepted 16 March 2006 